\title{
Research on the Information Sharing Between University Library and Booksellers Jiming Chu ${ }^{1}$, Ruheng Yin ${ }^{2}$ ${ }^{1}$ Library of Xuzhou University of Technology, Xuzhou, Jiangsu 221018;
${ }^{2}$ School of Arts of Xuzhou University of Technology, Xuzhou, Jiangsu 221008
}

\begin{abstract}
The main goal of this paper is to study how to strengthen the information sharing between University Library and the booksellers, improve their information decision system, and effectively improve the quality of book purchased. Through analysis of existing research results, this paper points out that the information sharing between University Library and the booksellers is bidirectional, mutually beneficial and trustful. It also points that they carry out information sharing in two aspects: information sharing main bodies and objects of information sharing behavior. At last, this paper proposes to strengthen the network construction of information sharing, improve the mechanism for resolving conflicts, establish fair principles of interest distribution, enhance the inclusiveness of organizational culture, and construct countermeasures of building information sharing such as strengthening trust system to promote the development of information sharing.
\end{abstract}

Keywords: University; Library; Library suppliers; Information sharing; Countermeasures

\section{高校图书馆与馆配商信息共享研究}

储济明 ${ }^{1}$ ，殷如恒 ${ }^{2}$

(1.徐州工程学院图书馆，江苏 徐州 221018；2.徐州工程学院艺术学院，江苏徐州 221008)

摘要: 主要目标是研究如何加强高校图书馆和馆配商的信息共享, 完善他们信息决策系统, 切实提高图书采购质量。通 过已有研究成果的分析, 指出高校图书馆和馆配商信息共享具有双向性、互利性、信任性的特征, 及他们在信息共享主体和 信息共享行为对象两方面开展信息共享的驱动力。提出应从加强信息共享网络构建、完善冲突解决机制、构建公平的利益分 配原则、提升组织文化的包容性、强化信任体系建设等方面构建信息共享的对策，推动信息共享工作的深入开展。

关键词:高校；图书馆；馆配商；信息共享；对策

中图分类号: G253.1 文献标识码: A

引言

高校图书馆与馆配商信息共享是指根据图书采购需要，经二者协商一致所构建起的信息共享渠道和共 享方式, 为双方的采购决策提供有效的信息保障。研究图书馆与馆配商的信息共享研究，对于促进双方相 互交流、理解、吸收、整合各自所拥有的信息 ${ }^{[1]}$ ，提高双方图示采购决策运行效率，为图书馆采购计划的 实现和馆配商经济效益的获取具有重要的意义和作用。

\section{1 高校图书馆与馆配商信息共享的驱动力}

对于高校图书馆和馆配商而言, 他们之间的信息共享驱动力主要来源于两方面: 行为主体和行为对象。 行为主体是指参与信息共享的高校图书馆和馆配商, 因他们在一定程度上具有共同的利益诉求, 从而驱使 他们主动开展信息共享。行为对象就是共享的信息, 即由于信息自身的特点, 促使高校图书馆和馆配商共 享信息，而且这种驱动力往往能在更深的层次上驱使高校图书馆和馆配商共享信息。 


\section{1 信息共享行为主体的驱动力}

\section{1.1 是完善各自信息系统的必然要求}

在信息社会, 信息已成为构建核心竞争力的核心要素。但对于高校图书馆和馆配商而言, 仅凭借自身 的努力, 都难以获取自己所需的全部信息, 信息不足已成为制约各自工作目标实现的主要因素 [2]。而通 过信息共享, 高校图书馆和馆配商可以识别、获取、吸收、使用和挖掘对方的信息, 将对方信息整合到自 己的信息系统中, 使得高校图书馆能充分掌握与图书采访有关的信息, 采购目标也越容易实现; 馆配商则 能更加准确地掌握高校图书馆需求信息, 把分散的高校图书馆需求信息整合到自己的信息系统中, 并借助 数据挖掘等技术对高校图书馆的需求信息进行分析, 逐步构建起不可模仿的和难以被超越的信息优势, 为 自身经济利益的充分实现提供有效的信息保障。因此说, 对于高校图书馆和馆配商而言, 信息共享已成为 完善各自信息系统，有效解决信息不足的问题，以提升决策效率，实现各自目标的必然举措。

\section{1.2 是降低采购成本的必然要求}

高校图书馆高校解决读者需求个性化需求不断增多、图书出版发行渠道多元化等因素给图书采购工作 带来的压力, 已成为其提高资金使用效率和图书采购质量所必须解决的问题。馆配商解决高校图书馆多样 化和个性化的需求, 对提高其业务水平和经济效益也具有重要影响。为了解决各自问题, 高校图书馆和馆 配商都不得不投入大量人力、物力、财力开展相关信息的搜集、加工、整理等工作，成本支出也在迅速增 加。而信息共享工作的开展, 就可以充分发挥高校图书馆和馆配商的信息搜集、整理、加工优势, 通力合 作, 有效避免信息搜集、加工、整理等工作中的重复投入, 切实降低采购成本支出, 实现高校图书馆与馆 配商双赢。

\section{1.3 是提高工作效率的必然举措}

在图书采购工作中，信息共享的开展，对于实现高校图书馆和馆配商业务流程协同，有效提高工作效 率具有重要的促进作用 [3]。信息共享不但可以使高校图书馆与馆配商在充分了解对方所拥有信息的基础 上开展决策，实现决策信息的协同。而且能有效提高图书馆需求信息与馆配商采访信息之间的契合度，提 高图书采购进度, 有效提升工作效率。

\section{2 信息共享行为对象的驱动力}

\section{2 .1 信息的异质性}

由于专业化社会分工的存在，高校图书馆和馆配商在图书采购工作承担不同角色，他们所拥有的信息 也呈现出明显的专业化、非均衡分布状态，在拥有同质信息的同时还拥有异质信息，而异质信息的存在就 使得在他们之间开展信息共享成为可能 [4]。因为异质信息的存在, 造成高校图书馆和馆配商的信息系统 均存在不完善之处, 信息共享则为他们获取对方异质信息来弥补各自的信息缺陷, 完善各自信息系统, 实 现科学决策成为可能。

\section{2.2 信息的外部依赖性}

随着图书出版渠道的多样化、馆配环境的变迁、高校图书馆需求的多样化等因素的影响，高校图书馆 和馆配商仅靠自身努力难以获取图书采购所需全部信息 [5], 他们都存在着信息的外部依赖性, 从外部获 取信息已经成为他们完善自身信息系统的重要举措。信息外部依赖性的存在就促使高校图书馆和馆配商不 得不参与信息共享, 唯有如此, 才能实现高校图书馆与馆配商之间的信息转移、整合与配置, 才能实现自 
身信息系统的完善，才能为各项工作的开展提供有效的信息支持。

\section{2 .3 信息匹配的流畅性}

图书采购的核心环节之一是图书馆需求信息和馆配商采访信息之间的匹配 [6]，而信息共享则是满足 这一匹配机制需求的最佳途径。通过信息共享能在高校图书馆和馆配商之间构建起一条有效的信息沟通机 制, 通过该机制, 一方面能实现满足匹配机制所需的信息能在高校图书馆和馆配商之间顺畅流动, 使他们 能最大限度地获取其所需要的信息, 另一方面能能切实提高馆配商书目数据与高校图书馆需求信息之间的 契合度, 从而确保信息匹配流畅性目标的充分实现。

\section{2 .4 信息价值实现的协作性}

信息作为一种重要的资源, 其价值往往需要通过相关部门的相互协作来实现, 协作性对于信息价值的 实现而言具有更为重要的意义和作用。信息价值实现的协作性就决定了在高校图书馆和馆配商之间必须开 展信息共享, 只有这样才能将他们所拥有的信息整合成为一个紧密的信息系统, 并通过他们共同协作, 最 大限度实现信息的价值。反之, 必然会因为高校图书馆和馆配商信息系统中的缺陷, 影响信息价值的实现 程度, 最终造成大量信息难以在其生命周期中发挥应有的作用。

\section{2 高校图书馆与馆配商信息共享的特征}

高校图书馆与馆配商之间虽然存在信息共享的驱动力, 但由于他们有着各自不同的利益诉求, 使得他 们之间的信息共享呈现出以下特征。

\section{1 双向性}

高校图书馆与馆配商之间的信息共享是一种双向互动的过程, 他们根据既定的目标, 同时承担着信息 提供者和信息接受者的角色，不但要将吸收、整合对方的信息，同时要把自己的信息提供给对方 ${ }^{[7]}$ 。

\section{2 互利性}

高校图书馆和馆配商信息共享的最终目标是实现双方共赢, 即高校图书馆能全面实现既定的采购目 标, 完善馆藏建设; 馆配商则能够提高销售额, 降低成本支出, 确保经济利益的充分实现。如果在信息共 享中不能实现互利, 而只有一方获利, 就必然会导致另一方放弃信息共享, 信息共享也就无法进行, 因此 说互利性实现信息共享的重要基础。

\section{3 信任性}

高校图书馆和馆配商之间信息共享的频度和深度主要由双方的信任度所决定 ${ }^{[8]}$, 信任度高, 双方共享 信息的主观意愿就越强, 信息共享就越容易实现; 信任度低, 双方就必然处于一种猜忌状态之中, 信息共 享的主观意愿必然不高, 信息共享也就无从谈起。

\section{3 高校图书馆与馆配商信息共享对策}

\section{1 加强信息共享网络建设}

信息共享网络是由多条信息共享链构成, 集信息共享、信息创造等功能为一体的网络体系 ${ }^{[9]}$ 。信息共 享链是指以参与信息共享的高校图书馆和馆配商为主体, 以实现信息共享和信息创造为目的, 通过信息在 参与信息共享与创新活动的高校图书馆和馆配商之间的流动而形成的链式结构。合理的信息共享网络和信 息共享链的构建, 为信息共享提供了一个有效的沟通渠道, 实现信息在高校图书馆和馆配商之间安全、合 理、快速流动和共享, 确保信息共享的充分实现。在构建信息共享网络和信息共享链时, 应充分考虑双方 
需求, 明确信息共享内容, 实现信息使用价值在参与共享的高校图书馆和馆配商之间的让渡, 使得双方对 信息共享所可能取得的成果形成一种现实的预期的前提下, 开展信息共享网络和信息共享链建设, 实现信 息共享。

\section{2 完善冲突解决机制}

由于高校图书馆和馆配商所追求的利益存在不同，造成他们在开展信息共享时，可能会因双方的利益 差异产生冲突, 阻碍信息共享的正常进行 ${ }^{[10]}$ 。为了避免这种情况的出现, 构建起完善的冲突解决机制, 及 时解决参与信息共享各方的冲突，就成为促进信息共享重要保障。在构建冲突解决机制时，应以互利共赢 为出发点, 以双向沟通、公平公正为基本原则, 确保该机制能在充分考虑参与共享各方利益的基础上, 为 公平解决共享各方的冲突提供解决方案, 以提高各方参加信息共享的主动性, 确保信息共享的长期深入进 行。

\section{3 构建公平的利益分配机制}

对于参与信息共享的馆配商而言, 能否实现他们对公平机会的追求, 建立并实施公平的利益分配机制, 对他们是否参与信息共享具有重大的影响 ${ }^{[11]}$ 。因此说, 为了给馆配商创造公平的信息共享环境, 构建起完 善的利益分配机制, 并以各方参与信息共享的程度为依据, 确定信息共享所产生的经济利益及因信息共享 所衍生出的新的信息资源和利益等的分配与归属原则，确保参与各方公平地分享信息共享成果，就成为提 高馆配商参与信息共享的主观愿望的重要措施。

\section{4 强化信任体系建设}

维系信息共享的因素除了高校图书馆和馆配商对信息的追求之外，最重要的因素就是相互之间的高度 信任，信任程度如何将在很大程度上决定信息共享实现的结果 ${ }^{[12]}$ 。因为信任作为高校图书馆和馆配商之间 的润滑剂, 它的存在对于降低信息共享中的机会主义行为, 避免对信息外溢, 构建良好的信息共享环境与 氛围, 促进信息共享深入进行具有重要作用。同时伴随着高校图书馆和馆配商之间信任度的不断提高, 信 息沟通渠道也会变得更加顺畅，信息共享程度也必然会随之加深。

\section{4 致谢}

感谢住房和城乡建设部 (项目编号：2015-K8-006) 和江苏高校哲学社会科学研究 (项目编号: 2012SJD870010) 基金的资助。

This work was supported by Ministry of Housing and Urban-Rural Development of the People's Republic of China (2015-K8-006) and universities' philosophy and social science researches in Jiangsu Provinc (2012SJD870010).

\section{参考文献:}

［1］胡平波,占峰波．知识共享中企业间认知差距维度结构及测量［J]．情报杂志，2011 (9)：139-144

[2］侯芳．基于信息资源优化配置理论的信息共享策略研究［J]．情报探索，2013(7) 75-77, 80

[3] 王学东, 谢辉, 谢晓娇. 面向知识共享流程的虚拟团队知识协同研究 [J]．情报科学，2011(11)：1608-1612

[4] 储济明. 非对称信息条件下馆配商激励措施研究 [J]．情报探索，2015(2)：101-103

[5] 王海花, 蒋旭灿, 谢富纪. 开放式创新模式下组织间知识共享影响因素的实证研究 [J]. 科学学与科学技术管理, $2013(6): 83-90$ 
[6] 刘菊霞. 提升资源建设与用户需求契合度的基本路径: 以馆配商采访书目的精细化为例. 出版发行研究, 2016(8) : 94-97

[7] 李久平, 顾新. 知识联盟组织之间知识共享研究 [J]. 情报杂志, 2007 (7) : 91-93

[8] 黄昱方, 郑文杰. 跨边界情境下关系嵌入影响知识团队知识转移机制研究 [J]. 情报科学, 2012(2)：190-194

[9] 陈健, 顾新, 吴绍波. 知识网络公平感对知识共享的影响及路径研究 [J]. 情报杂志, 2011(4):107-112, 106

[10］周杰, 张卫国. 战略联盟企业间动态关系与知识转移：联盟管理能力的调节作用 [J]. 科技进步与对策, $2013(2): 1-5$

[11］杨淑琼. 高校图书馆与馆配商合作机制探讨 [J]. 图书馆学研究，2016(7):33-37

[12］黄昱方, 郑文杰. 跨边界情境下关系嵌入影响知识团队知识转移机制研究 [J]. 情报科学, 2012 (2) : 190-194

\section{References:}

[1] Hu Pingbo, Zhan Fengbo. Dimension Structure and Measurement on the Perception Gap of Knowledge-sharing between Enterprises [J]. Journal of Intelligence, 2011 (9): 139-144

[2] Hou Fang. Research of Information Sharing Strategy Basing on Information Resource Optimized Collocation Theory [J]. Information Research, 2013 (7) 75-77,80

[3] Wang Xuedong, Xie Hui, Xie Xiaojiao. Research on Knowledge Sharing Process-oriented Knowledge Collaboration in Virtual Team [J]. Information Science, 2011 (11):1608-1612

[4] Chu Jiming. Study on Incentive Measures for Book Suppliers under the Condition of Asymmetric Information [J]. Information Research, 2015(2):101-103

[5] Wang Haihua, Jiang Xucan, Xie Fuji. Empirical Study on the Influential Factors of Inter-Organizational Knowledge Sharing Under Open Innovation Model [J]. Science of Science Management of S. and T., 2013 (6): 83-90

[6] Liu Juxia. The Basic Way to Improve the Compatibility between the Construction of Resources and the Needs of Users: A Case Study on the Refinement of the Bibliography of Booksellers. Publishing Research, 2016 (8):94-97

[7] Li Jiuping, Gu Xin. A Study on Knowledge Sharing among Organizations in Knowledge Alliance [J]. Journal of Intelligence, 2007 (7): 91-93

[8] Huang Yufang, Zheng Wenjie. Research on Effect of Relational Embeddednesss on Knowledge Transfer in Knowledge Teams in Circumstances of Cross Boundary [J]. Information Science, 2012 (2):190-194

[9] Chen Jian, Gu Xin, Wu Shaobo. Influence The Path of Knowledge Network's Justice Perception Influencing Knowledge Sharing [J]. Journal of Intelligence, 2011 (4): 107-112106

[10]Zhou Jie, Zhang Wei. Dynamic Relationship and Knowledge Transfer in Strategic Alliances: Moderating Role of Alliance Management Capability [J]. Science and Technology Progress and Policy, 2013 (2): 1-5

[11] Yang Shuqiong. Discussion on Cooperation Mechanism between University Libraries and Library Suppliers [J]. Research on Library Science, 2016 (7): 33-37

[12]Huang Yufang, Zheng Wenjie. Study on Effect of Relational Embeddedness on Knowledge Transfer in Knowledge Teams in Circumstances of Cross Boundary [J]. Information Science, 2012 (2):190-194 